\title{
The Raz Bladder Suspensions: Treatment of Stress Urinary Incontinence: 10 Years Personal Experience
}

\author{
Ernest H. Agatstein ${ }^{*}, 1$ and Shlomo Raz ${ }^{2}$ \\ ${ }^{I}$ Department of Urology, Geffen School of Medicine at UCLA, USA \\ ${ }^{2}$ Division of Pelvic Medicine and Reconstructive Surgery, Geffen School of Medicine at UCLA, USA
}

\begin{abstract}
Introduction: We report on one surgeon's clinical experience with the 2 and 4 corner Raz bladder suspension performed for the treatment of stress urinary incontinence (alone or associated with cystocoele repair) in a consecutive patient cohort between 2003 and 2012 reporting short and longer term results.

Materials and Methods: We performed a retrospective study of consecutive patients who underwent a 2 or 4 corner Raz bladder suspension by one surgeon (EHA ) to treat stress urinary incontinence over a period of time from Jan 12003 -Sept 2012. 3 month and longer term outcomes was determined by patient self assessment including validated questionnaires.

Results: $98.7 \%$ of 82 patients reported no stress incontinence at 3 months Longer term follow-up was available on 48 $(65 \%)$ patients. Of those lost to follow up, 8 were deceased. Mean age was 66.1 years. Mean follow up was 48.2 months (range $4-111$ months). 21 of the 48 (44\%)patients were beyond 48 months. 41 of 48 (85\%) patients were either delighted, pleased or mostly satisfied. 42 of 48 patients $(87.5 \%)$ had $50 \%$ or more improvement. Pad use of 2.6 decreased to 0.9 pads with $P$ value less than 0.0001 .
\end{abstract}

Conclusion: The Raz bladder suspension has good short and longer term results in relieving stress incontinence in addition to low morbidity and lower costs without the use of any mesh product.

Keywords: Stress, treatment outcome, urinary incontinence.

\section{INTRODUCTION}

The surgical management of stress urine incontinence (SUI) has undergone significant change over the last 40 years. In the decades before 1980, the predominant method of surgical repair were the retropubic suspensions(MarshallMarchetti or Burch). Dr. Shlomo Raz in the early 1980's introduced the modified Peyrera needle suspension resulting in the 2 and 4 corner Raz bladder suspensions so widely used and reported on [1-8]. There have been no recent reports on the Raz suspensions.

The purpose of this article is to demonstrate the short and longer term effectiveness of the Raz procedure in the treatment of SUI. One of the authors(EHA), has performed the 2 and 4 corner Raz suspensions both as stand alone procedures and combined with vaginal hysterectomy, anterior and posterior repair. Adoption of electronic medical records in 2003 has made retrospective review of the last 10 years of surgical experience possible. The Raz procedure, a vaginal procedure without the use of mesh which suspends the bladder neck in a fixed retropubic position using nonabsorbable suture to create a vaginal hammock similar to the retropubic Burch suspension but using the rectus fascia as opposed to Coopers ligament as the anchor for the suspending sutures, may be a viable alternative for those

*Address correspondence to this author at the West Coast Urology, $575 \mathrm{E}$. Hardy St. Suite 215, Inglewood, California 90301, USA; Tel: 3106733333; Fax: 3106731714; E-mail: agatern@aol.com surgeons wishing to avoid the implantation of mesh in their repairs.

\section{MATERIALS AND METHODS}

We performed a retrospective study on all patients operated on by a single surgeon (EHA) who underwent a Raz bladder neck ( 2 corner) or bladder suspension( 4 corner suspension) procedure between June 2003 and May 2012. Preoperative evaluation for each patient included history, pad use, physical examination, cystoscopy, Marshall testing(with $200 \mathrm{cc}$ in bladder patient asked to cough or valsalva with urine leaking per urethra as a positive Marshall test) and 2 channel cystometrogram to determine Abdominal leak point pressure.

Inclusion criteria was any patient with subjective SUI or patients documented objectively to have leakage with Valsalva after reduction of cystocele if present. Exclusion criteria were patients with no subjective SUI and no objective demonstration of leakage with Valsalava even with reduction of cystocele if present.

The following systematic approach was used. 2 corner suspension was performed when no cystocoele was identified on exam. A 4 corner suspension was performed in the presence of a Grade 1 cystocele based on the BadenWalker classification with mainly lateral defect. Patients with Grade 2 cystocele (anterior wall at the hymen with strain) or Grade 3 cystocele(anterior wall beyond the hymen 
with strain) or any cystocele of any degree with midline defect underwent formal cystocele repair performed by gynecology combined with Raz suspension performed by the urologist. The cystocele repair was usually completed with a pursestring method or classic Kelly plication via midline incision. After the vaginal wall was closed in the midline, the Raz incisions in the lateral sulcus on both sides were made and either 2 corner or 4 corner suspension was performed depending on how much prolapse remained after the repair. We found that repairing the cystocele and closing the vaginal mucosa in the midline and then making separate incisions for the Raz repairs facilitated the lateral dissection and exposure of the retropubic space and allowed easier placement of the suspending sutures. In cases of uterine prolapse, a vaginal hysterectomy was performed by gynecology with cystocele repair if needed followed by the Raz procedures performed by the urologist. Rectocele was repaired if present by gynecology in standard posterior repair.

The Raz procedures were performed as illustrated in the textbook Atlas of Transvaginal Surgery $2^{\text {nd }}$ Edition (Raz) [9]. Number 1 prolene sutures were used to take 3 helical bites of the vaginal wall excluding the vaginal epithelium and the margin of the urethropelvic fascia until one could move the patient with the sutures under traction. 2 sutures were used for the 2 corner repair and 4 sutures ( 2 on each side) for the 4 corner suspension. Intraoperative cystocopy was performed after intravenous indigo carmine to identify blue from the ureteral orifices and to identify any Prolene sutures inside the bladder.

All patients received antibiotic prophylaxis. Patients had their Foley catheter removed within 24 or 48 hours. Those unable to void had a Foley reinserted. Patients underwent voiding trial at 1 week. After 2 weeks of urine retention patients were taught intermittent self catherization.

Retrospective office chart review was carried out on consecutive patients operated on between June 2003 and May 2012 and data systematically recorded. Age at surgery, preoperative ALPP, number of preoperative pads used per 24 hour, presence of postoperative urine retention or other postoperative or intraoperative complications, presence or absence of SUI at 3 months postoperative were reviewed and recorded.

Validated patient self assessment questionnaires were mailed by a third party who was not the treating urologist to all the above patients. Efforts were made to contact by telephone all missing patients. Family members informed us if the patient was deceased. Outcome was determined by patient self assessment. The questionnaires included the validated symptom questionnaires that assessed the amount of bother, the validated short form of the Urogenital Distress Inventory(UDI-6) and the presence or absence and frequency of symptoms, the validated Incontinence Symptom Score (ISS-8) $[10,11]$. Scores were reported as 0-not at all, 1slightly, 2-moderate and 3-greatly [12]. Questions related to quality of life due to urinary symptoms, pad use, overall improvement of urinary condition and improvement rating score were also included [12, 13] The PGII (Patient Global Impression of Improvement) questionnaire asked to check the number that best describes how the patients post operative condition is now, compared with how it was before surgery(1-very much better 2-Much better 3-a little better 4- no change 5-a little worse 6-much worse and 7-very much worse). The Quality of Life Score (QOL) was assessed-(If you were to spend the rest of your life with your urinary condition just the way it is now, how would you feel-0-delighted 1-pleased 2-mostly satisfied 3-mixed 4-mostly dissatisfied 5-unhappy 6-terrible). An assessment of protection was determined by asking-(How many pads do you use in 24 hours?) The improvement score was assessed by asking-(compared to before surgery, how much in percent have you improved ?) Each patient completed all of these multiple and varied validated assessment tools.

Patient survey data on the longer term cohort was tabulated with number of months elapsed since surgery, UDI-6 bother score, ISS-8 score, PGI-1 score, Quality of life score, percent improvement, number of pads worn preoperative and at time of survey. Unpaired t test was used to compare pre and postoperative pad use using QuickCalcs and Microsoft Excel 2007. All patients were informed of their inclusion in a retrospective study at the time the questionnaires were sent

\section{RESULTS}

A total of 82 patients underwent either a 2 or 4 corner Raz procedure between 6/4/2003 and 5/17/2012. 23 patients who had SUI and no cystocele underwent a 2 corner suspension only. 25 patients who had SUI and Grade 1 cystocoele underwent a 4 corner suspension only without formal cystocele repair. 13 patients with Grade 2 or worse cystoceles underwent formal anterior/posterior repair along with a Raz suspension ( 9 patients underwent 2 corner Raz and 4 patients underwent 4 corner Raz). 11 of these 13 had subjective complaints of SUI. 21 patients underwent a combined procedure with vaginal hysterectomy and anterior/posterior repair (17 of these underwent 2 corner Raz and 4 underwent 4 corner Raz). 16 of these 21 had subjective complaints of SUI.

Mean age was 62.8 years(range 30-86). Mean ALPP was $82 \mathrm{~cm}$ of water (range $30-140$ ). Mean pre-operative pad use per 24 hours was 2.7 (range 0-8). There were no intraoperative bladder or ureteral injuries identified and no postoperative blood transfusions. 36 patients had postoperative urine retention( $43 \%)$ and were sent home with their foley catheter. 24 patients $(29 \%)$ had retention for 1 week only.(Clavien Grade 1 complication). 7(8\%) patients had retention for 2 weeks, 1 for 3 weeks, 3 for 4 weeks and 1 for 6 weeks. No long term retention needing urethrolysis was encountered.

81 of the 82 patients $(98.7 \%)$ reported no SUI at the 3 month postoperative visit. Nine patients $(10.9) \%$ reported symptoms of urgency at 3 month needing antimuscarinic therapy, all with low postvoid residuals. 4 patients had postoperative UTI $(4.8 \%)$ (Clavien Grade 2). 5 patients $(6 \%)$ had small wound separations in the first 1-2 weeks postoperative all with negative cultures with complete healing. One patient had continued SUI at 3 months and was diagnosed with ISD.

Longer term results were available on 48 patients.; 8 were deceased at time of survey, 26 could not be located resulting in longer term data on 48 of the available 74 patients $(65 \%)$. Of these 48 patients, 12 had a combined 
Table 1. Long term cohort data table.

Mean number of months post surgery survey conducted= 48.2 months (range 4-111 months) SD 32.11

\begin{tabular}{|c|c|c|c|c|c|c|c|c|c|}
\hline \multicolumn{3}{|c|}{ Results } & \multicolumn{3}{c|}{ Means (Standard Deviation) } \\
\hline \# of pts & $\begin{array}{c}\text { Age at Surgery } \\
\text { (Range) }\end{array}$ & $\begin{array}{c}\text { Age at Survey } \\
\text { (Range) }\end{array}$ & PGII Score & Qol Score & UDI 6 & ISS & \% Improved & Preop Pads & Postop Pads \\
\hline \hline 48 & $63.4(40-83)$ yrs & $66.1(45-91)$ yrs & $2.1(1.3)$ & $2.0(1.8)$ & $7.6(6.7)$ & $7.42(6.44)$ & $69.4(28.8)$ & $2.62(1.69)$ & $0.93(1.24)^{1}$ \\
\hline
\end{tabular}

vaginal hysterectomy/cystocele repair(10 patients with 2 corner and 2 patients with 4 corner), 8 underwent formal cystocoele repair( 6 patients with 2 corner and 2 patients with 4 corner), 12 had a 2 corner suspension only and 16 had a 4 corner suspension only. A total of 28 patients underwent 2 corner Raz suspension and 20 patients underwent 4 corner Raz suspesnion. The mean age at time of surgery was 63.47 (range 40-83). The mean ALPP was 87.9 (range 40-140). Only 4 of 48 patients ( $8 \%$ ) had ALPP less than 60 . The mean patient age at time of survey was 66.1 years (range 45-91). Mean number of months postoperative when surveys were completed was 48.2 months (range 4-111 months). 21 of 48 $(43.7 \%)$ patients were beyond 48 months. Patient demographics and self assessment tools results are summarized in Table $\mathbf{1}$. There were no significant demographic differences between the patients who underwent 2 corner suspension $v s 4$ corner suspension.

The mean PGI-1 score was 2.1 ( $2=$ much better). 36 of the $48(75 \%)$ patients were very much or much better. If score 3 which represents "a little better" is included as a treatment success, then 41 of 48 patients $(85 \%)$ would have met the criteria for treatment success. The mean Quality of life score was $2.0(2=$ mostly satisfied $) 41$ of $48(85 \%)$ were delighted, pleased or mostly satisfied with their postoperative condition. 52\% were delighted or pleased. Mean percent improvement was 69\% (range 0-100). 42 of 48 patients $(87.5 \%)$ reported $50 \%$ or more improvement. Preoperative pad use for this cohort was 2.6 (Range 0-6) Postoperative pad use was O.9 (range 0-4). This difference was statistically significant at a $\mathrm{P}$ value less than 0.0001 .26 of $48(54 \%)$ patients reported wearing 0 pads per day. $70 \%$ of patients reported using less pads There were no statistical differences in all of the outcome measures when we compared the cohort who underwent 2 corner suspension with the patients who underwent 4 corner suspension.

The UDI- 6 and ISS- 8 self assessment data are reported as raw distribution score and mean score (Table 2). The mean UDI-6 bother score was 7.62. Using Question 3 of the UDI $6,77 \%$ of patients reported either not at all or only slightly being bothered by SUI. The mean ISS symptom score was 7.4. Using ISS question 5 for SUI, 75\% of patients reported SUI symptoms as none or less than once per week.

\section{DISCUSSION}

In 1981, Raz described modifications of the Peyrera bladder neck suspension [1]. It was felt that this procedure was best suited for patients with anatomic incontinence due to urethral and bladder neck hypermobility with minimal or no cystocoele. In the Raz suspension, the support of the bladder neck and bladder is on the midline suprapubic area just above the pubic bone not into the muscle but rather on the fascial attachment to the periosteum. Many authors have reported on the success of the Raz bladder neck suspension procedure [2-8]. A prospective cohort of over 400 patients at UCLA based on objective and subjective criteria revealed a cure rate of $85 \%$ [5]. These studies showed the effectiveness of the Raz bladder neck suspension in the treatment of SUI in patients with little or no cystocoele. The 2 corner bladder neck suspension was modified by Raz to a 4 corner bladder and bladder neck suspension in patients with grade 2 and 3 cystocoeles who had concomitant SUI. Raz reported on 120 patients with Grade 2 and 3 cystocoeles who underwent 4 corner suspension with 2 year mean follow up with $94 \%$ of the patients having relief of their SUI [6].

The Raz and other needle suspensions fell out of use in the last decade by the majority of practicing urologists. Papers were published showing decreased benefits over time of the Raz procedure [14-16]. The 1997 AUA Guidelines (Leach et al.) [17] showed a lower cure dry rate at 48 months with transvaginal needle suspensions (67\%) compared with retropubic suspensions $(84 \%)$ or pubo-vaginal autologous slings $(83 \%)$. That review panel considered transvaginal needle suspensions to be" a good option for the appropriate women with SUI who are willing to accept worse long term benefit in favor of lower immediate morbidity".

The autologous fascial pubovaginal sling procedures reported by Mcguire and Blaivas [18, 19] showed excellent long term results but had increased morbidity with the need to harvest rectus fascia. When the TVT and other types of mid or distal urethral slings using synthetic materiel were introduced, conceptually they had the same benefits as an autologous fascial pubo-vaginal sling without the morbidity of harvesting fascia. The adoption of midurethral sling suspensions by urologists were as a result of their being minimally invasive without the need to enter the retropubic space, being easy to teach and perform. The Cochrane data base in 2004 reviewed the role of needle suspensions for SUI and concluded that although they were more likely to fail than open retropubic suspensions, the evidence was limited: no conclusions could be drawn when compared to the subrethral sling [20].

There have been no recent published series of the short and longer term success rates of the Raz suspensions. Our current study documents that in one individual surgeons hands, the Raz 2 and 4 corner suspensions have excellent success at relieving SUI at 3 months and acceptable longer term success rates with mean post-operative follow up of 48 months in the range of $75-85 \%$ across a wide range of subjective metrics with low complication rates and without 
Table 2. Post Operative self assessment symptoms (mean follow up 48 months).

\begin{tabular}{|c|c|c|c|c|c|c|c|}
\hline & & \multicolumn{6}{|c|}{ Number of Patients Reporting Score (\%) } \\
\hline Incomplete emptying & 1 & $23(52)$ & $9(20)$ & $7(16)$ & $5(11)$ & 0.88 & 0.16 \\
\hline Urgency & 2 & $22(50)$ & $16(36)$ & $3(7)$ & $3(7)$ & 0.69 & 0.12 \\
\hline Daytime frequency & 4 & $18(41)$ & $14(32)$ & $11(25)$ & $1(2)$ & 0.88 & 0.13 \\
\hline Stress incontinence & 5 & $24(55)$ & $9(20)$ & $3(7)$ & $8(18)$ & 0.90 & 0.17 \\
\hline Urge Incontinence & 6 & $21(47)$ & $12(27)$ & $3(7)$ & $8(18)$ & 0.95 & 0.17 \\
\hline Leakage level of activity & 7 & $25(57)$ & $6(14)$ & $9(20)$ & $4(9)$ & 0.83 & 0.16 \\
\hline Urge incontinence & 2 & $21(44)$ & $11(23)$ & $8(17)$ & $8(16)$ & 1.06 & 0.16 \\
\hline Stress incontinence & 3 & $25(52)$ & $12(25)$ & $3(6)$ & $8(16)$ & 0.91 & 0.16 \\
\hline Small leakage amount & 4 & $22(46)$ & $13(27)$ & $7(15)$ & $6(12)$ & 0.97 & 0.15 \\
\hline Difficult emptying & 5 & $32(67)$ & $6(13)$ & $4(8)$ & $6(12)$ & 0.69 & 0.15 \\
\hline Pain & 6 & $32(67)$ & $8(16)$ & $6(12)$ & $2((4)$ & 0.52 & 0.12 \\
\hline Night time urination & 7 & $14(29)$ & $15(31)$ & $13(27)$ & $6(12)$ & 1.20 & 0.14 \\
\hline Urgency & 8 & $20(42)$ & $13(27)$ & $6(12)$ & $9(19)$ & 1.13 & 0.16 \\
\hline
\end{tabular}

the use of any mesh product. Only $14 \%$ of patients required catheterization longer than 1 week. No patient suffered long term urine retention requiring urethrolysis. No intraoperative bladder or ureteral injuries were recognized and no transfusions used. No complaints of dyspareunia and no exposures or erosions of prolene sutures were encountered at least in the first 3 months. There are other potential benefits of the Raz suspensions. The midline placement of the suspension sutures avoids the ilioinguinal nerve that can be involved with performance of retropubic procedures like the TVT or Sparc. The Raz suspensions can easily be performed in combination with vaginal hysterectomy and cystocoele/rectocoele repairs in patients with significant pelvic prolapse. Although our study looks at both 2 and 4 corner suspensions and many were done in combination with vaginal hysterectomy and cystocele repairs, all of these patients had some degree of stress urine incontinence(overt or occult) which is unlikely to have improved without an associated procedure designed to treat stress urinary incontinence. Our study focuses on the ability of the Raz suspensions to solve the issue of SUI whether the SUI presents as the only issue, whether it is present with associated moderate cystocele or whether it is present along with more severe degrees of pelvic organ prolapse. Our results showed no statistical differences in SUI outcome regardless whether we performed a 2 vs 4 corner Raz suspension.

The Raz bladder neck suspensions are native tissue repairs without the use of mesh. Early complications associated with mesh such as pelvic pain, erosions and later complications ( $8-10$ years) due to vaginal atrophy and aging of the tissues are potential reasons why the pelvic surgeon may want to avoid the use of mesh and consider the Raz suspensions as an alternative.

Limitations of our study include not using validated questionnaires preoperatively. $16-18 \%$ of our longer term cohort reported severe urge incontinence. Some of these patients may have had this urge incontinence preoperatively however some of these patients may represent de novo urgency as a post operative complication. These patients were managed by behavioral techniques and antimuscarinic therapy. No patient was found to be severely obstructed to require a urethrolysis at any time in the period surveyed. $43 \%$ of patients had some degree of urine retention in the immediate post-operative period although no permanent retention was seen. Although no objective exam or clinical testing was performed in the postoperative assessment of the longer term cohort as part of this study, the consistent results across multiple, different validated subjective self assessment tools adds credibility to our outcome data. A significant number of our original cohort was lost to follow up. While only $65 \%$ of the available cohort were assessed long term, the demographics, ALPP and preoperative pad usage between the initial complete cohort and the longer term cohort were similar.

Since 1987, this surgeon(EHA) continuously performed 2 and 4 corner Raz suspensions resisting changing technique because of good results achieved. The AUA Guidelines, issued in 2009 for surgical management of SUI (Appel et al.) stated "the Panel did not review needle suspensions in 
developing the guideline. Though these operations may still be performed in isolated circumstances by some surgeons, the panel believes they are of largely historical interest". We submit that in light of the data reported in this article, the Raz suspensions today may be more than just of historical interest but rather of significant use in the armamentarium of the practicing urologist.

\section{CONCLUSION}

This article demonstrates that the Raz two and four corner bladder suspensions performed both as a stand alone procedure or combined with other repairs of vaginal prolapse to relieve patients with SUI has acceptable short and longer term success rates. This procedure may be a viable alternative to urologists when considering options for the surgical management of SUI.

\section{CONFLICT OF INTEREST}

Agatstein is a speaker for Astellas. No conflict of interest. Raz has no financial disclosures. No conflict of interest.

\section{ACKNOWLEDGEMENTS}

We acknowledge the participation of Dr. Emanuel Mba as the operating gynecologist on the majority of our joint prolapse cases as well as UCLA undergraduate senior, Joshua Hekmatjah for his assistance.

The authors have no financial or any other conflicts of interest. The research was a retrospective chart review of patients already treated, done without patient identifiers and conducted according to the Helsinki guidelines. All persons gave informed consent before being included in the study.

Dr. Ernest Agatstein Protocol and Project development /data collection and management/data analysis/manuscript writing and editing

Dr. Shlomo Raz Protocol and project development/data analysis/manuscript writing and editing.

\section{Project Tasks}

Agatstein-Protocol/project development/data collection and management data analysis/ manuscript writing /editing.

Raz-protocol/ project development/data analysis/editing.

\section{REFERENCES}

Raz S. Modified bladder neck suspension for female stress incontinence. Urology 1981; 17: 82-5.
[2] Nitti VW, Bregg KJ, Sussman EM, Raz S. The Raz bladder neck suspension in patients 65 years and older. J Urol 1993; 149(4): 8027.

[3] Raz S, Sussman EM, Erickson DB et al. The Raz bladder neck suspension: results in 206 patients. J Urol 1992; 148(3): 845-50.

[4] Golomb J, Goldwasser B, Mashaich S. Raz Bladder neck suspension in women younger than 65 compared with elderly women: three years experience. Urology 1994; 43(1): 40-3.

[5] Raz S, Strothers S, Chopra A. Raz Techniques for Anterior Vagina 1 Wall repair. Female Urology, Second edition, Ch 30, , Philadelphia, Saunders 1996; p. 344.

[6] Raz S, Klutke CG, Golomb J: Four corner bladder and urethral suspension for moderate cystocoele. J Urol 1989; 142: 712-5.

[7] Pastor SF, Cisnak MJM, Chicote PF, et al. Treatment of stress incontinence in women by the Raz technique: results. Arch Espan Urol 1992; 45(1): 59-61.

[8] Naudin M, Hauzeur C, Schulman C. Raz method of bladder suspension and treatment of cystocoele in urinary stress incontinence (short term results). Acta Urol Belg 1992; 60 (2): 6775.

[9] Raz S. Transvaginal Needle Bladder Neck Suspension and Four Corner Bladder and Urethral Suspension For Moderate Cystocoele, Atlas Of Transvaginal Surgery, Ch 5, Philadelphia, Saunders, 2002; pp. 76-86, pp. 127-32.

[10] Uebersax JS, Wyman JF, Shumaker SA et al. Short forms to assess quality and symptom distress for urinary incontinence in women: the Incontinence Impact Questionnaire and the Urogenital Distress Inventory. Continence Program for Women Research Group. Neurourol Urodyn 1995; 14: 131-9.

[11] Twiss $\mathrm{C}$, Triaca $\mathrm{V}$, Anger $\mathrm{J}$ et al. Validating the incontinence symptom severity index: a self assessment instrument for voiding symptom severity in women. J Urol 2009; 182: 2384-91.

[12] Rodriguez LV, Raz S. Prospective analysis of patients treated with a distal urethral polypropylene sling for symptoms of stress urinary incontinence: surgical outcome and satisfaction determination by patient driven questionnaires. J Urol 2003; 170: 857-63.

[13] Rutman M, Itano N, Deng et al. Long term durability of the distal urethral polyprpylene sling procedure for stress urinary incontinence: minimum 5 year followup of surgical satisfaction determined by patient reported questionnaires. J Urol 2006; 175 : 610-13.

[14] Trockman BA, Leach GE, Hamilton J, et al. Modified Peyrera bladder neck suspension: 10 year mean follow up using outcomes analysis in 125 patients. J Urol 1995; 154: 1841-7.

[15] Korman IU, Sirls LT, Kirkemo AK: Success rate of modified Peyrera bladder neck suspension determined by outcomes analysis. J Urol 1994; 152: 1453-7.

[16] Masson DB, Govier FE. Modified peyrera bladder neck suspension in patients with intrinsic sphincter deficiency and bladder neck hypermobility: patient satisfaction with a mean follow up of 4 years. Urology 2000; 55: 217-21.

[17] Leach GR, Domchowski RR, Appel RA, et al. Female stress urinary incontinence guidelines panel summary report on surgical management of female stress urine incontinence. J Urol 1997; 158: 875-80.

[18] McGuire EJ, Bennet CJ, Konnak JA, et al. Experience with pubo vaginal slings for urinary incontinence at University of Michigan. J Urol 1987; 138: 525-6.

[19] Blaivas JG, Jacobs BZ. Pubovaginal fascial sling for the treatment of complicated stress urinary incontinence. J Urol 1991; 145: 12148.

[20] Glaezner CM, Cooper K. Bladder neck needle suspension for urinary incontinence in women. Cochrane Database Syst Rev 2004; 2: CD003636. 